\title{
Image analysis of ceramic burning based on cellular automata
}

Xiaowei $\mathrm{Gu}^{1^{*}}$ and Yiqin Sun ${ }^{2}$

\begin{abstract}
In order to effectively improve the flexural strength and fracture toughness of ceramics, two methods can be used to control the growth rate of grain during sintering of ceramic materials by adding a certain amount of (W,Ti)C material. Therefore, on the one hand, the paper simulates the composite ceramic materials based on cellular automata (CA) to optimize the ratio of two formulations. On the other hand, in order to optimize sintering process, CA modeling is also carried out for the sintering process of composite ceramics. Finally, in order to detect the temperature of the firing zone of the ceramic kiln by using the characteristics of the flame image of the firing zone, the K-means clustering method is used for the color segmentation of the flame image of the firing zone of the ceramic kiln. The experimental results show that the size of the grains is in accordance with the actual situation of the simulation and the microstructure evolution of the composites can be simulated well by using the CA theory to simulate the composites; with the increase of simulation time, the grain size distribution is basically unchanged, which accords with the normal distribution, and the simulation process of grain growth is very stable. Based on the K-mean clustering segmentation method, the segmentation of the flame image of ceramic kiln firing zone is realized. This method also provides a good technical means for feature extraction of flame image in ceramic kiln firing zone.
\end{abstract}

Keywords: CA, Composite ceramic materials, Ceramic burning image

\section{Introduction}

Ceramic material has high hardness, high wear resistance, and good high temperature stability, as well as good electrical and thermal conductivity and chemical resistance [1]. Therefore, there is a great prospect in the field of refractories, nozzles, cutting tools, and bearings. In addition, ceramic material belongs to the boron-containing series ceramics and can generate boron oxide lubricating film dissolved with other elements under friction and high temperature $\left(800-1000{ }^{\circ} \mathrm{C}\right)$ driving, which can realize self-lubricating and is a very good self-lubricating material [2]. Ceramic material has good neutron control capabilities and can be used in the nuclear industry. However, the sintering densification of ceramic materials is more difficult in bending strength, and the defects of low fracture toughness limit its application scope. It often fails because

\footnotetext{
* Correspondence: gxwsyq1980@163.com

Xiaowei Gu and Yiqin Sun contributed equally and are co-authors of this manuscript.

${ }^{1}$ School of Information Science and Technology, Zhejiang Sci-Tech University, 928 Second Avenue, Xiasha Higher Education Zone, Hangzhou, Zhejiang,

People's Republic of China

Full list of author information is available at the end of the article
}

of damage [3]. Related studies have shown that [4] (W,Ti)C composites can effectively increase the bending strength and fracture toughness of composite ceramic materials. With the increasing demand for composite materials in modern engineering, during the preparation of composite ceramic materials, if the formulation is not optimized, ceramic material grain growth and abnormal growth may occur during the preparation process. (W,Ti)C grain distribution is not uniform and will not achieve the purpose of improving the performance of composite ceramic materials. Therefore, optimizing the proportion of the two components in composite ceramic materials is very important to improve the performance of composite ceramic materials. In addition, adding (W,Ti)C composites to ceramic material can also significantly improve its mechanical properties. The level of mechanical properties of [5] is closely related to the microstructure. But the mechanical properties are closely related to the microstructure. The grain growth during sintering determines the evolution of the microstructure and the local topological structure of the sintered body, and the bending strength of the sintered body. Fracture toughness and 
hardness play an important role. Therefore, it is of great significance to study the grain growth in the sintering process of ceramic materials and optimize the sintering process to improve the bending strength, fracture toughness, and hardness of the materials.

In recent years, many studies have been carried out on the grain growth in the process of preparing materials by computer simulation. The commonly used research methods are [6]: phase field method, Monte Carlo method, and so on. However, the models also have some defects, such as the low efficiency of [7] Monte Carlo method, the complexity of phase field theory model, the large amount of calculation, and the gap between [7] Monte Carlo method and the actual model. In 1991, Hesselbarth et al. [8] firstly used CA method to simulate the kinetics of recrystallization nucleation and grain growth of materials and obtained the same results as that of JAMK theory. With the increasing of simulation research on grain growth based on CA method, Liu [9] proposed a two-dimensional grain growth model combining MC method and CA method. Geiger et al. [10] provided the CA method based on thermodynamics which is proposed to simulate the two-dimensional growth of grains. Ding [11] et al. established two-dimensional and three-dimensional CA models based on the minimum energy principle. Raghavan et al. [12] proposed a small increment processing method for cells. Guan Xiaojun et al. [13] and Ma Xiaofai et al. [14] also simulated the grain growth by CA method. CA shows complex behaviors through cellular interaction through simple operation rules. It has become the main tool for modeling complex systems. However, the application of CA method to the simulation of grain change in the sintering process of composite ceramic materials is relatively rare.

In the process of industrial production, the sintering zone temperature of ceramic kiln is an important factor affecting the firing quality of ceramic products, so it is very necessary to study the advanced detection and control methods of ceramic kiln firing belt condition. In recent years, with the development of image processing technology, most of the industrial production control through the extraction of flame characteristics of the analysis of the state of material combustion. Effective flame segmentation is one of the key techniques to detect temperature by flame image, so it is necessary to study the effective flame image segmentation technology. The common image segmentation methods include threshold method and clustering analysis. The threshold method is suitable for gray image segmentation, but not for flame image color segmentation. $\mathrm{K}$-means clustering algorithm is a mature clustering analysis method, which has been successfully applied to face image segmentation and remote sensing image analysis and other fields.
Therefore, this paper starts with two aspects. On the one hand, CA theory is used to simulate the composite materials, and the relationship between grain distribution and microstructure is analyzed. It is of great significance to select the preparation process reasonably, save the experiment cost, shorten the experiment period, and improve the experiment efficiency. On the other hand, using CA theory, the CA model of grain growth during sintering of composite ceramic materials is established. The grain growth of ceramic materials was simulated. The relationship between grain size distribution and simulation time was analyzed, and the growth index was calculated and compared with the prepared ceramic materials. In addition, in the Lab color space, the K-mean clustering is applied to the segmentation of the flame image of the combustion zone of the ceramic kiln and the experiment is carried out. Finally, the segmentation and contrast experiments are carried out in different color modes.

\section{Method-establishment of CA model \\ 2.1 CA model}

CA can be considered to be composed of many grids. Each cell in the grid is a cell and only takes a finite discrete state. All of them follow the same local transformation rules and update [15] synchronously. A large number of cells form the dynamic evolution of the system through simple interaction and can form a complex evolutionary state. CA [16] is not defined by a definite function, but is determined by simple rules. That is, CA model is the general name of dynamic system which satisfies certain rules. The states of each variable are only limited, and the rules of state change are local.

Cells, cellular spaces, neighbors, and rules make up a CA. The most basic unit of CA is a cell, which is located on a discrete grid of one-dimensional, two-dimensional, or multidimensional spaces. The state of a cell may consist of $\{0 \sim 1\}$, or it may be a discrete set in the form of an integer [17]. The space dot set of cellular distribution is called cellular space, and its geometric partition can be Euclidean space partition of arbitrary dimension. One-dimensional and two-dimensional CA are the focus of current research. One-dimensional CA has only one form of cell space partition, and two-dimensional cell space division is very flexible. It can be arranged in triangle, square, or hexagonal grid [18], as shown in Fig. 1. The (a) diagram in Fig. 1 is a cell space partition form of one-dimensional CA. The (b), (c), and (d) diagrams are the cell space partition forms of two-dimensional CA, which are the triangle, square, and hexagon, respectively.

The cellular space can be extended infinitely in every dimension, which is helpful to the theoretical study, but this ideal condition cannot be realized in practical application. Therefore, it is very important to define different 


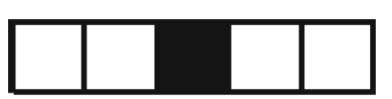

a Grid arrangement of one dimensional cell

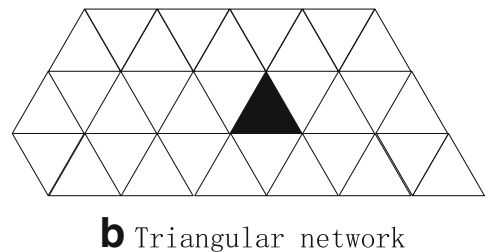

b Triangular network

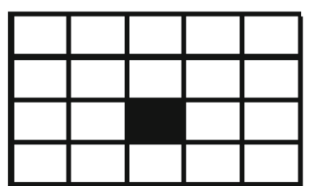

C Square network

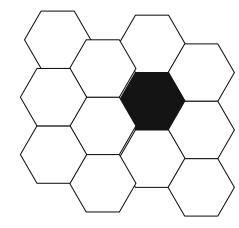

hexagonal network

Fig. 1 a-d One-dimensional and two-dimensional CA space partitioning

boundary conditions. There are generally three types of boundary conditions: reflection type, fixed value type, and periodic type [19]. In some cases, random forms can be used to simulate real-time natural phenomena, that is, to generate random numbers at the boundary in real time.

The cellular space and the cellular space represent only the static components of the system, and the dynamic system is formed by adding the evolution rules to the system. These rules in CA are defined in a local space, and a cell will determine the state of the next moment according to its own state and the state of its neighbor's cell; therefore, the definition of neighbor rules is the premise of CA operation, and it must be clear which one cell neighbor has. One-dimensional CA can determine neighbors according to radius. Two-dimensional CA defines neighbors more complex, and there are two general ways: One is a von Neumann neighbor, where the cell's neighbors are composed of four cells adjacent to each other from the top, bottom, left, and right. The radius is 1 . The other is the Moorish neighbor, where the cell neighbors are composed of upper, lower, left, right, upper, lower right, lower left, and lower left cells [20].

The rule is the dynamic basis of CA evolution and the dynamic function to determine the state of the cell at the next moment, that is, [21].

$$
f: S_{i}^{t+1}=f\left(S_{i}^{t}, S_{N}^{t}\right)
$$

where $f$ denotes the evolution rule of the state and $S_{i}^{t}$ denotes the state of the cell $I$ at the $t$ moment and the state, while $S_{N}^{t}$ represents the state of the neighbor cell of the cell $I$ at the time of $t$.

\subsection{Simulates the composite ceramic materials based on CA (simulation of grain structure evolution) \\ 2.2.1 The physical significance of the model}

Grain boundary energy is the driving force of grain growth, which is closely related to the curvature of grain boundary. The movement of grain boundary is determined by the change of grain boundary energy and curvature of grain boundary. If the grain boundary energy is isotropic, the grain boundary energy $E_{i}$ of cell $I$ can be expressed as [22].

$$
E_{i}=J_{i}^{k} \sum_{k}^{n n}\left(1-\delta_{S_{i}} \delta_{S_{k}}\right)
$$

where $J_{i}^{k}$ is a measure of the grain boundary energy between the cell $i$ and the adjacent $k$, the total number of the adjacency of $n$ is the cell $i . \delta$ is the orientation of the Kronechaer symbol, $S_{k}$ being the adjacent $k$. If the orientation value of the cell $I$ is changed to the orientation value of the cell $j$, then the change of the grain boundary energy is recorded as $\Delta E_{i, j}$, that is, [23].

$$
\Delta E_{i, j}=E_{j}-E_{i}
$$

\subsubsection{Simulation of grain structure evolution}

The simulation process of grain structure evolution consists of four main steps: discrete simulation space, initialization of lattice points, presupposition of parameters, and determination of evolution rules.

\section{- Discrete simulation space}

In this paper, a two-dimensional square cell space is used to divide the simulated region, and the adjacent relation is of the Moore-Moore-Moore type, as shown in Fig. 2. At this time, the neighbor of the cell is composed of eight cells located in the upper, lower, left, right, upper, lower and lower left, and the lower left of the eight adjacent positions.

\section{- Initialization lattice}

The initial lattice is to initialize the state of all the cells. At the beginning of the simulation, each cell is given a random orientation value. If the orientation value of the adjacent cell is the same, it will be 


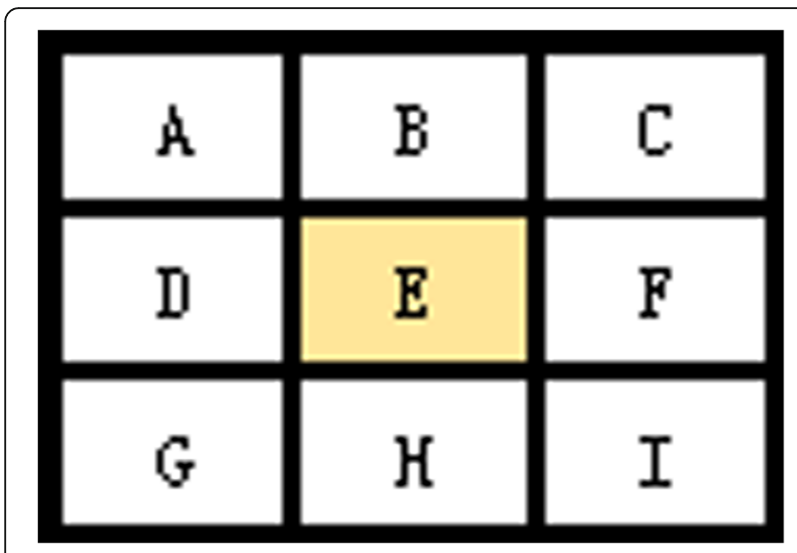

Fig. 2 Neighbor definition of Moore type for two-dimensional cellular automata

regarded as the same particle, and if different, it will be regarded as different grain. The state of the cell $E$ is expressed as [24].

$$
\begin{aligned}
E(t)= & f(A(t-\Delta t), B(t-\Delta t), C(t-\Delta t), D(t-\Delta t), E(t-\Delta t), \\
& F(t-\Delta t), G(t-\Delta t), H(t-\Delta t), I(t-\Delta t))
\end{aligned}
$$

where $\Delta t$ is the time step.

- Prediction parameters

The prediction parameter is to give a predetermined value to each parameter in the simulation process.

- Deterministic evolution rule

The evolution rules used in this paper contain four conditions:

(1) If the state of cell $E$ at $t$ is the same as that of eight adjacent cells, then the state of $E$ remains unchanged in the next time step.

(2) If any three of the nearest neighbor cells of cell $E$ are states $a$ at $t$, the state of $E$ will change to $a$ at the next time step.

(3) If any three of the next nearest neighbor cells of cell $E$ are states $b$ at $t$, the state of $E$ changes to $b$ in the next step.

(4) If the above conditions are not satisfied, a cell is randomly selected from the eight adjacent cells, and the grain boundary of the system can change $\Delta E_{i, j}$ after calculation. According to the transition probability $P$, the change of the cell is determined, and $P$ uses the following reorientation probability model:

$$
P=\left\{\begin{array}{cc}
1 & \Delta E_{i, j}<0 \\
0.5 & \Delta E_{i, j}=0 \\
0 & \Delta E_{i, j}>0
\end{array}\right.
$$

In each time step of the simulation, the order of the transition of cellular orientation state is as follows: condition 1 , condition 2 , condition 3 , and condition 4 . When one of the conditions is satisfied, the corresponding orientation state transition is carried out, and the subsequent judgment steps can be omitted. Finally, all the other cellular orientation state transitions are judged in turn, and a time step of the cell state transition process is completed.

In this paper, the initial modeling is carried out according to the material performance parameters shown in Table 1, and the simulation is carried out according to the content of each component in Table 2. The whole process includes discrete simulation space, initialization of lattice points, presupposition of parameters, and determination of evolution rules, programming, simulation, and result analysis.

\subsection{Carry out CA modeling for sintering process of composite ceramics}

In order to optimize the sintering process, a CA model of grain growth in the sintering process of composite ceramics was established, which can simulate the grain growth of ceramic materials. The growth index is solved by analyzing the relationship between grain size distribution and simulated time. Generally, the CA modeling process of sintering process of composite ceramics mainly includes determining the initial conditions of simulation, determining the unit grain boundary energy, and pore initialization.

\section{- Determining the initial conditions of simulation}

In this paper, a quadrilateral mesh structure of $600 \times 600$ is used, and periodic boundary conditions are used. The actual size of the side length of each cell is $0.12 \mu \mathrm{m}$. A

Table 1 Each component performance parameters in the ZrB2/ (W,Ti)C composites

\begin{tabular}{lllll}
\hline Material & $\begin{array}{l}\text { Density } \\
\left(\mathrm{g} / \mathrm{cm}^{3}\right)\end{array}$ & $\begin{array}{l}\text { Thermal } \\
\text { expansion } \\
\text { coefficient }\end{array}$ & Poisson's ratio & $\begin{array}{l}\text { Modulus of } \\
\text { elasticity (GPa) }\end{array}$ \\
\hline $\mathrm{Z}_{\mathrm{r}} \mathrm{B}_{2}$ & 5.2 & 6.21 & 0.21 & 550 \\
$(\mathrm{~W}, \mathrm{Ti}) \mathrm{C}$ & 9.13 & 5.5 & 0.3 & 553 \\
$\mathrm{ZrO}_{2}$ & 6.12 & 9.3 & 0.25 & 200 \\
\hline
\end{tabular}


Table 2 Proportion of composition of composite materials

\begin{tabular}{llll}
\hline Sample name & $\mathrm{V}((\mathrm{W}, \mathrm{Ti}) \mathrm{C})(\%)$ & $\mathrm{V}\left(\mathrm{Z}_{\mathrm{r}} \mathrm{B}_{2}\right)(\%)$ & $\mathrm{V}\left(\mathrm{Z}_{\mathrm{r}} \mathrm{O}_{2}\right)(\%)$ \\
\hline ZW0 & 0 & 93.3 & 6.7 \\
ZW0 & 5 & 88.3 & 6.7 \\
ZW10 & 10 & 83.68 & 6.32 \\
ZW15 & 15 & 78.68 & 6.32 \\
ZW20 & 20 & 73.8 & 6.2 \\
ZW25 & 25 & 65.8 & 9.2 \\
ZW30 & 30 & 62.4 & 7.6 \\
ZW35 & 35 & 58.4 & 6.6 \\
ZW40 & 40 & 52.4 & 7.6 \\
\hline
\end{tabular}

random orientation number is given to each cell before the simulation. The orientation number of ceramic matrix is in the range of $1001 \leq S_{i}^{1} \leq 1500$, and the orientation number of other particles is in the range of $1 \leq S_{i}^{2} \leq 1000$, which represents the value of each cell; crystallographic orientation of different cells in grains. Two percent of sintering auxiliaries were used as inert particles to give a fixed orientation value. After the ratio of composite materials to sintering auxiliaries was set, the program was randomly distributed in the matrix. Here, it is assumed that the sintering auxiliaries neither migrate nor react and diffuse with other phases during the simulation process.

- Determining the unit grain boundary energy

In the two ball densification model of simple cubic stacking, the relationship between two angles and grain boundary energy is

$$
J_{s s}=2 J_{s v} \cos \frac{\phi}{2}
$$

In formula: $J_{\mathrm{SS}}$ is the grain boundary energy $(J)$ between solid phase and solid phase, $J_{\mathrm{SV}}$ is the grain boundary energy $(J)$ between solid phase and gas phase, and $\phi$ is the angle of two sides.

- Pore initialization

The average size of stomata can be determined by the following formula.

$$
d_{a}=\frac{2 D_{a}(1-f)}{3 f}
$$

In formula, $d_{a}$ is the average diameter of pores $(\mu \mathrm{m})$, $D_{a}$ is the average diameter of powder particles $(\mu \mathrm{m})$, and $f$ is the density of plain billet. In the simulated initialization structure, the porosity is $20 \%$, then $f=0.8$. According to formula (7), $D_{\mathrm{a}}=0.29 D_{a}$ can be obtained. It is necessary to initialize the structure first and then add the stoma to initialize the hole at 0.29 $D_{a}$.

\subsection{Image segmentation of ceramic kiln flame based on K-means clustering}

The K-means clustering algorithm is iterative implementation of image classification and extraction of all kinds of eigenvalues. Its basic ideas are as follows: First, the average value of each class is first removed, then the pixels are reclassified according to the newly generated mean, and then the previous steps are iterated to the new generated class. In this paper, the K-means clustering algorithm based on criterion function is used for flame image segmentation. The general process consists of two steps:

\subsubsection{Color space conversion}

First, transform the RGB color space image into Lab color space. The purpose of image segmentation is to separate the target part. Based on the K-means clustering segmentation method, the segmentation of the flame image of ceramic kiln firing zone is realized. This provides a good

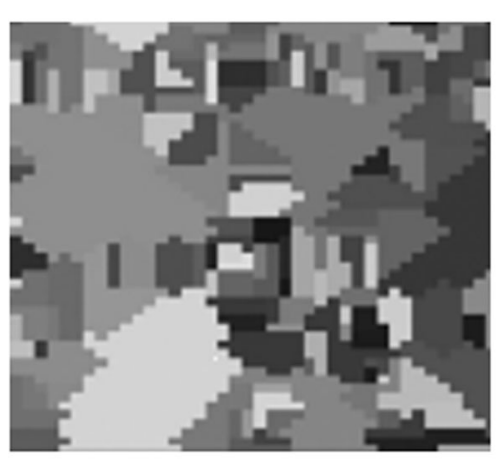

a ZW10

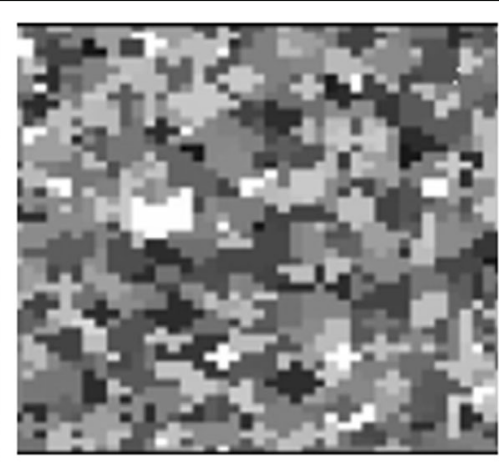

b ZW30

Fig. $\mathbf{3}$ a, $\mathbf{b}$ Simulation of microstructure of ZW series composite ceramic materials 
technical means for feature extraction of flame image in ceramic kiln firing zone distributed into simple geometry. Therefore, the flame can be separated from the image by K-means clustering according to the color information. The commonly used color space models are HSV, RGB, and Lab. Among them, the Lab color model is a color model made by the CIE (International Lighting Committee). Its color space is larger than the RGB space and can be expressed in all colors in nature. Therefore, this paper converts the RGB color space to XYZ color space. Then, the $\mathrm{XYZ}$ color space is converted to Lab color space.

\subsubsection{Flame image segmentation using $K$-means clustering}

The general steps are as follows:
(1) Initialize. Any $K$ attribute value vector is taken as the center of the initial clustering. According to the maximum cycle number or the convergence error tolerance principle of the cluster center, the iteration termination condition is set.

(2) Iteration. According to the similarity criterion, the data objects are assigned to the nearest cluster center, and $K$ clustering data sets are obtained.

(3) Updated cluster center. The average value of each point in the cluster dataset is calculated, and then, the average value of each class is used as the new clustering center to reassign the data object.

(4) Judge whether clustering is completed. If the clustering center is no longer changed or the sum

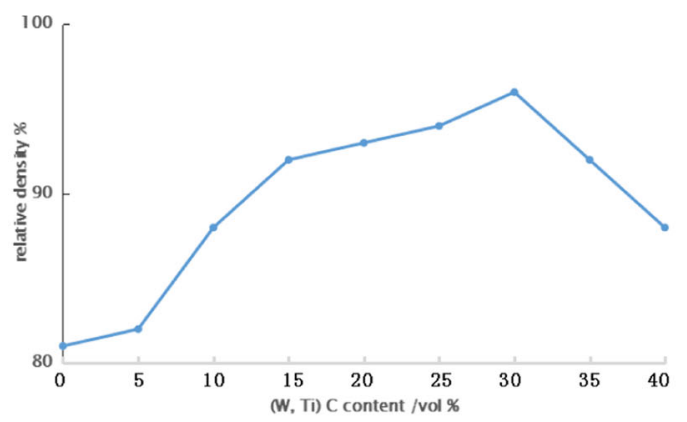

a Relationship between $(\mathrm{W}, \mathrm{Ti}) \mathrm{C}$ content and relative density

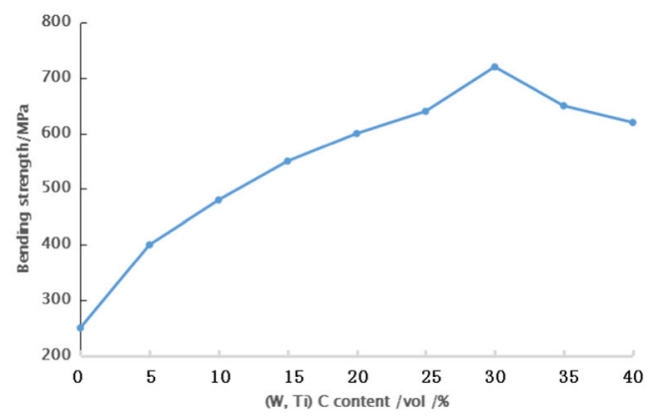

b Relationship between (W,Ti)C content and bending strength

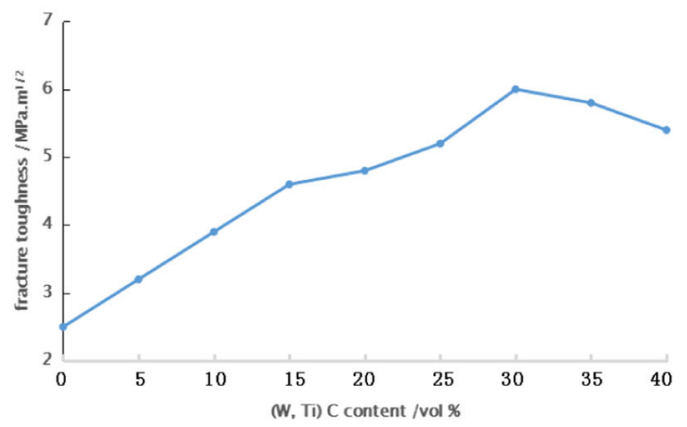

C Relationship between $(\mathrm{W}, \mathrm{Ti}) \mathrm{C}$ content and fracture toughness

Fig. 4 The curves of the a relative density, $\mathbf{b}$ bending strength, and $\mathbf{c}$ fracture toughness of ceramic materials with the content of TI-C 


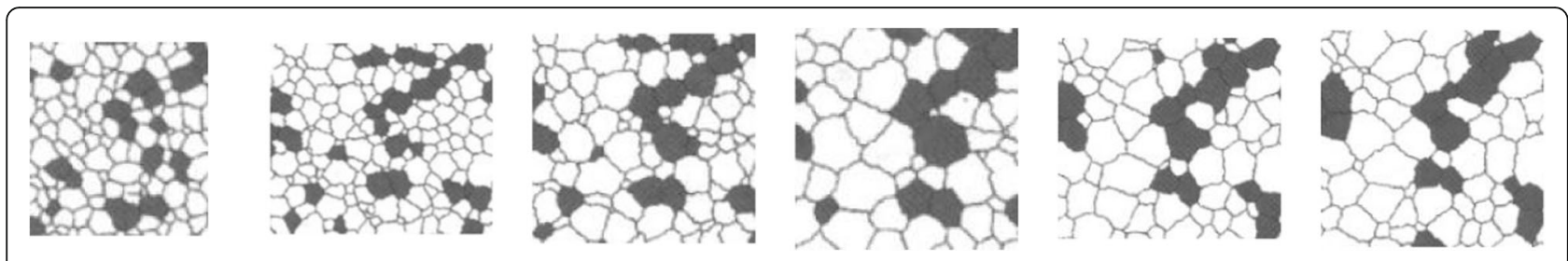

Fig. 5 Microstructure evolution of grain growth from time to time from 500 to 2500 CAS

of square errors is local minimum, then the iteration is terminated and the clustering segmentation is completed. Otherwise, the second and third steps are repeatedly executed until the suspension condition is satisfied.

\section{Experimental results and discussions \\ 3.1 CA simulation experiment of composites \\ 3.1.1 Experiment}

In this paper, three kinds of materials with different proportions of $\mathrm{Z}_{\mathrm{r}} \mathrm{B}_{2} /(\mathrm{W}, \mathrm{Ti}) \mathrm{C} /\left(\mathrm{Z}_{\mathrm{r}} \mathrm{O}_{2}\right)$ are used, as shown in Table 1. The raw materials of $\mathrm{Z}_{\mathrm{r}} \mathrm{B}_{2}$ and $(\mathrm{W}, \mathrm{Ti}) \mathrm{C}$ were respectively milled in anhydrous ethanol for $50 \mathrm{~h}$, then dried at $150{ }^{\circ} \mathrm{C}$ for $24 \mathrm{~h}$, and then sifted in a vacuum dryer to obtain more uniform fine powder. The primary refined $\mathrm{Z}_{\mathrm{r}} \mathrm{B}_{2}$ was used as the matrix material, the strengthening phase, $\mathrm{Z}_{\mathrm{r}} \mathrm{O}_{2}$, was used as the toughening phase, and the addition of $\mathrm{MgO}$ sintering aid was carried out. The prepared raw materials were poured into a beaker with anhydrous ethanol as the medium, and the ultrasonic wave was oscillated and stirred for $30 \mathrm{~min}$ for prevention of material agglomeration. The mixture was milled with anhydrous ethanol for $200 \mathrm{~h}$ and dried in a vacuum drying box at $150{ }^{\circ} \mathrm{C}$ for $24 \mathrm{~h}$. The homogeneous fine powder suitable for sintering was obtained. The mixed raw material powder was put into the high strength graphite mold, and the hot pressing sintering process was adopted in vacuum environment.

\subsubsection{Comparison of results between $Z W 10$ and $Z W 30$}

The simulation of microstructure of ZW series composite ceramics is shown in Fig. 2. From Fig. 3, it can be seen that when the content of $(\mathrm{W}, \mathrm{Ti}) \mathrm{C}$ increases gradually, the grain size decreases, the grain boundary becomes smooth and straight, the grain number is increasing, and the grain boundary length in the simulated region decreases gradually. From the grain shape, the grain turns from larger cells to three sides. In contrast, the grain size of ZW30 is obviously smaller than the grain size of ZW10, and the distribution is more uniform, mainly because the proportion of ZW30 $(\mathrm{W}, \mathrm{Ti}) \mathrm{C}$ is more reasonable.

\subsubsection{Comparison of relative density, flexural strength, and fracture toughness of ceramic materials with different proportions}

Figure 4 shows the curves of the relative density, flexural strength, and fracture toughness of $\mathrm{ZW}$ series ceramic materials with the change of (W,Ti)C content.

It can be seen from Fig. 4 that the density, bending strength, and fracture toughness of ZW30 composite ceramics are the highest. The density, flexural strength, and fracture toughness of ZW0 composite ceramics are increased by 13.58 and $132 \%$, respectively, so the ratio of ZW30 composite ceramic materials is the best.

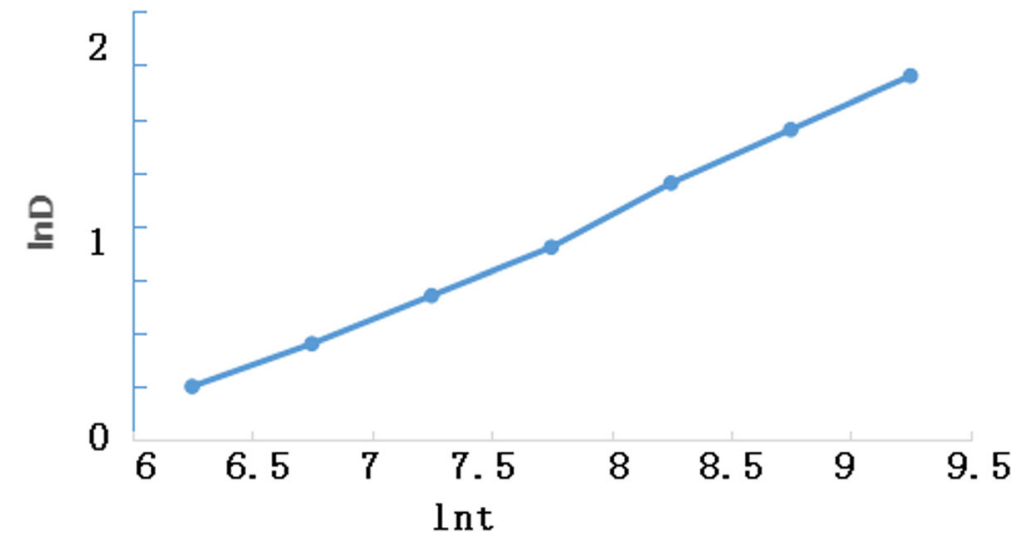

Fig. 6 Logarithmic relationship between average grain diameter and simulated time 


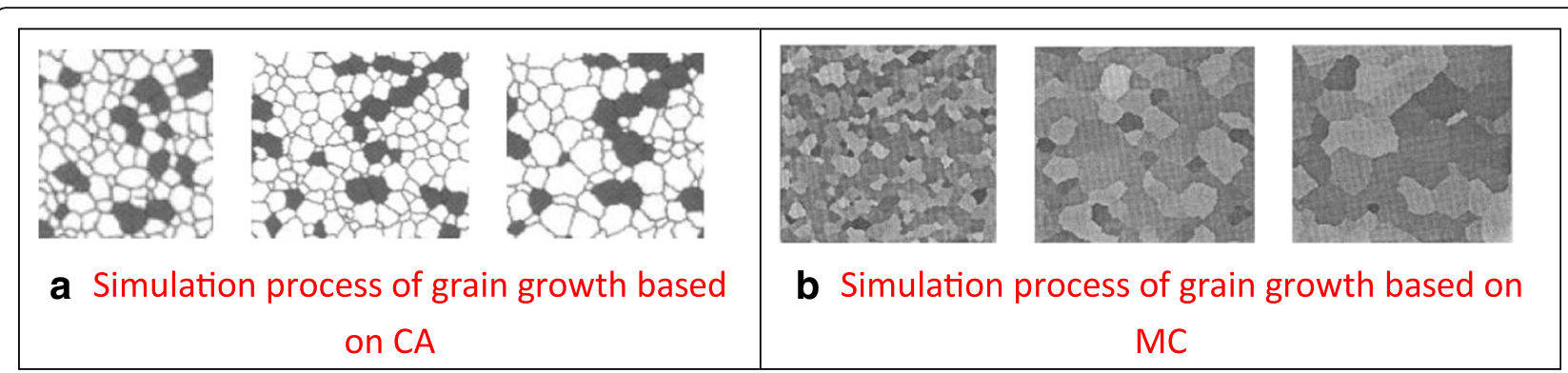

Fig. 7 Comparison between a CA method and $\mathbf{b}$ MC method for simulating grain growth process

\subsection{CA simulation of grain growth during sintering of composite ceramics \\ 3.2.1 Experiment}

The simulation object is the grain growth of $Z_{r} B_{2}$ / (W,Ti)C with $6.32 \% \mathrm{Z}_{\mathrm{r}} \mathrm{O}_{2}$ particles during sintering. According to the formula (5), at the initial stage of sintering, because the two balls did not penetrate, the two sided angle phi $=0, \mathrm{JSS} / \mathrm{JSV}=3$. The ratio of unit grain boundary energy is $J_{S 1 S 1}: J_{S 2 S 2}: J_{S 1 V}=2: 3$ : $3: 1$. First, give the matrix a random orientation value.

The composite ceramics and sintering additives were randomly added to the matrix according to the set proportion. After running $300 \mathrm{CAS}$, the average grain diameter reached about $0.85 \mu \mathrm{m}$ and the porosity was initialized. At this time, $40 \%$ of the grains were randomly selected according to the number of grains, and the orientation value was assigned to 1501, which was defined as porosity. Because of the different grain size, the area of the simulated area is about $32 \%$, that is, the density of the green is 0.68 . Figure 4 is the microstructure image of composite ceramics with simulated time step. The white grain is $\mathrm{Z}_{\mathrm{r}} \mathrm{B}_{2} /(\mathrm{W}, \mathrm{Ti}) \mathrm{C}$ grain, the gray grain is $\mathrm{ZrO} 2$ grain, the dark gray square grain is sintering aid, the light gray line is grain boundary, and the black area is pore.
It can be seen that the adjacent pores are through, and the distribution state is very similar to the distribution of pores in the billet.

\subsubsection{Comparison of grain size distribution in different time steps}

Figure 5 shows the grain size distribution of different simulated time steps. When the time step is $500 \mathrm{CAS}$, the distribution of grain size is relatively stable, the number of grains of each size is normal distribution, the grain size of middle size is the majority, the grain size of smaller size is very small, and the case of abnormal grain growth is also rare. With the increase of simulation time, the grain size distribution is basically unchanged, and all of them accord with the normal distribution, which shows that the grain growth process is very stable.

\subsubsection{Variation of average grain diameter with simulated time}

The curvature driven grain growth satisfies the following equation

$$
\bar{R}_{g}=(k t)^{n}
$$

where $n$ is the grain growth index, the shear slope of the $\ln R g$-lnt curve is obtained. The grain size in this paper is characterized by the diameter $D_{a}$. As

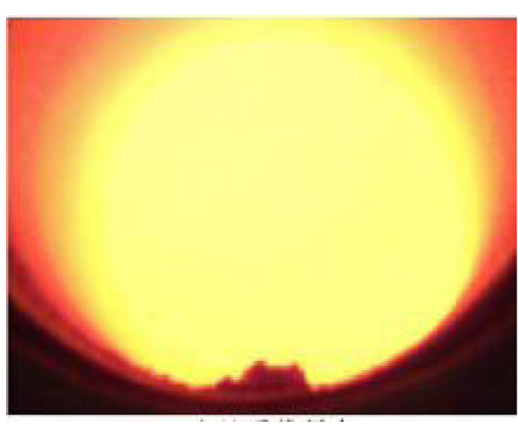

a Original flame image

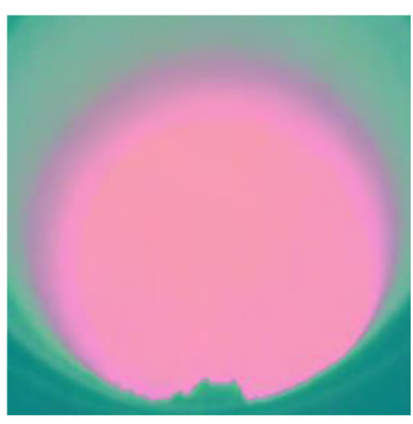

b Lab color space image

Fig. 8 Contrast of $\mathbf{a}$ original image to $\mathbf{b}$ Lab color space 


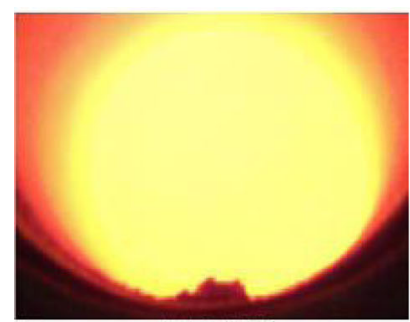

C Original flame image

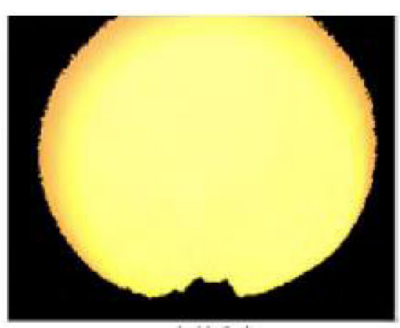

d Divided area 1

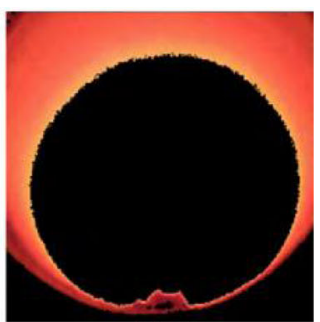

e Divided area 2

Fig. 9 c-e Clustering segmentation results

$$
\frac{D_{a}}{2}=(k t)^{n}
$$

As shown in Fig. 6, the logarithmic diagram of grain mean diameter with simulated time shows that the logarithmic diagram of grain mean diameter with time is basically a straight line, and its slope is 0.82 . It shows that the growth index simulated in this paper is slightly lower. The average grain size obtained in the simulation process may be slightly smaller than that of the real microstructure.

\subsubsection{The comparison between CA method and MC method} In order to verify the performance of CA method applied to the simulation of grain growth in the sintering process of composite ceramics, CA method and MC method are used to simulate the grain growth in the sintering process of composite ceramics under the same conditions. The simulation results are shown in Fig. 6, in which the time step is from 500 to 1500 CAS.

As can be seen from Fig. 7, the grain size distribution in (a) diagram is more stable than that in (b) diagram. The number of grains of various sizes is normal distribution, the majority of grains are of middle size, and the few grains of smaller size and the abnormal growth of grains are very few. And with the increase of simulation time, the grain size distribution in the (a) diagram is basically unchanged, which is in good agreement with the normal distribution. And the crystal particles in (b) diagram are still increasing slowly. It can be seen that the grain growth process in (a) diagram is very stable. Therefore, the effect of CA simulation is more stable than that of $\mathrm{MC}$ simulation in the simulation of grain growth in the sintering process of composite ceramics.

\subsection{Flame image segmentation in ceramic sintering process}

According to the characteristics of ceramic kiln flame image, the RGB color space image is first converted into Lab color space. The picture effect is shown in Fig. 8. Finally, according to the experimental results and experimental results, the K-means clustering segmentation of ceramic kiln flame images has achieved good results when $K$ and $n$ are taken as 3, as shown in Fig. 8. According to the K-means clustering method of Fig. 9, the image of ceramic kiln fired with flame is segmented well, and every part of the image is well distinguished after the segmentation.

In order to quantify the segmentation results, PRI (Probabilistic Rand Index), VOI (variation of information), GCE (Global Consistency Error), and BDE (Boundary Displacement Error) are used to analyze and evaluate the segmentation results from different perspectives. PRI is the ratio of the total number of pixels to the total number of pixels between statistical machine segmentation and manual separation. VOI is used to measure the distance between two segmented images, indicating the randomness of the segmented image relative to manual segmentation. GCE is used to measure the consistency of segmented images compared with manual segmentation. BDE measures the edge average error of two segmented images, which is defined as the distance between a pixel on the edge of the automatic segmentation and the nearest pixel of the artificially segmented image. In this experiment, 10 flame images with different air volume are selected and segmented by Otsu, K-means, FCM, and MCWT, respectively. The mean and standard deviations of PRI, VOI, GCE, and BDE of each segmentation method are calculated, and the time required for segmenting the flame image is also calculated. The performance of the segmentation algorithm is evaluated objectively. The comparison results are shown in Table 3 and Fig. 10.

As can be seen from Table 3 and Fig. 10, Otsu segmentation time is the shortest, but its segmentation accuracy is too low to provide reliable information for flame detection. The K-means segmentation time is

Table 3 Comparison of time required for segmentation of flame images using different algorithms

\begin{tabular}{lllll}
\hline Time & Otsu & K-means & FCM & MCWT \\
\hline Max & 0.004 & 0.602 & 6.942 & 2.198 \\
Min & 0.002 & 0.124 & 2.331 & 1.181 \\
Average & 0.003 & 0.320 & 4.213 & 2.101 \\
\hline
\end{tabular}



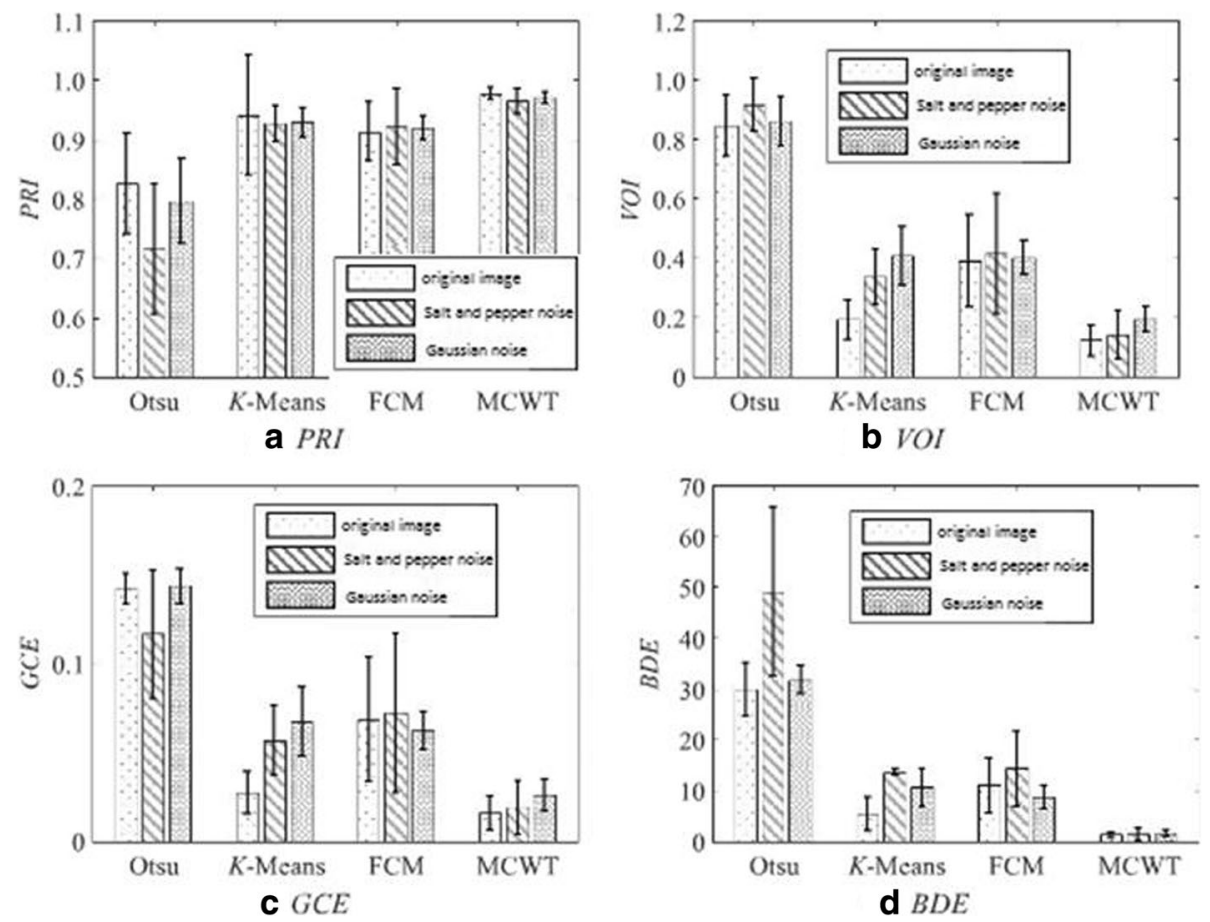

Fig. 10 a-d Four evaluation indexes of flame image segmentation algorithm

relatively short, the PRI of the algorithm is larger, and the VOI and BDE are smaller. It shows that the K-means algorithm has higher accuracy and better stability. The segmentation time is relatively short. Therefore, combined with various evaluation indexes, the K-means algorithm has the best segmentation effect.

\section{Conclusions}

It is difficult to densify its flexural strength, and its application range is limited by its low fracture toughness, so as to improve the flexural strength, fracture toughness, and hardness of $\mathrm{Z}_{\mathrm{r}} \mathrm{B}_{2}$ ceramic material. In order to improve the flexural strength, fracture toughness, and hardness of $\mathrm{Z}_{\mathrm{r}} \mathrm{B}_{2}$ ceramic material, it can be added to $\mathrm{Z}_{\mathrm{r}} \mathrm{B}_{2}$ ceramic material by adding a certain proportion of $(\mathrm{W}, \mathrm{Ti}) \mathrm{C}$ and optimizing sintering process. However, with the addition of (W,Ti)C and how to optimize the sintering process of composite ceramic materials, the CA theory is used to simulate the composite materials and the relationship between grain distribution and microstructure is analyzed in this paper. Choose the best proportion of composite ceramic materials. The grain growth in the sintering process of composite ceramic materials was also established based on long CA model. The grain growth of ceramic materials was simulated. The relationship between grain size distribution and simulation time was analyzed, and the growth index was calculated. Finally, in Lab color space, the K-means clustering is used to segment the flame image of the combustion zone in ceramic kiln and the experiment is carried out. Finally, a comparison experiment is carried out under different color modes. Through simulation experiments, it is found that:

(1) With the increase of (W,Ti)C content, the morphology of the grains continues to evolve. The grain size of ZW30 is obviously smaller than that of $\mathrm{ZW} 10$, and the distribution is more uniform, which is mainly due to the reasonable proportion of (W,Ti)C in ZW30 formula, and the grain size of ZW30 is smaller than that of ZW10, and the grain size of ZW30 is smaller than that of ZW10, and the grain size of ZW30 is more uniform than that of ZW30.

(2) The density, flexural strength, and fracture toughness of ZW30 composite ceramic material were improved by $13.58,160$, and $132 \%$, respectively.

(3) With the increase of simulation time, the grain size distribution is basically unchanged, which accords with the normal distribution, and the simulation process of grain growth is very stable. The simulated grain growth index $n$ is 0.42 .

(4) Based on K-means clustering method, the image segmentation of ceramic kiln fired with flame is presented.

\section{Abbreviations}

BDE: Boundary Displacement Error; CA: Cellular automata; CIE: International Lighting Committee; GCE: Global Consistency Error; HSV: Hue saturation value; PRI: Probabilistic Rand index; RGB: Red green blue; VOI: Variation of information 


\section{Acknowledgements}

The authors thank the editor and anonymous reviewers for their helpful comments and valuable suggestions. I would like to acknowledge all our team members, especially Yiqin Sun.

\section{Funding}

This research was supported by the National Natural Science Foundation of China under grant no. 51407156, Public welfare Technology Application Research Programs of Science and Technology Department of Zhejiang province under grant no. 2016C33018, and the Project Grants 521 Talents Cultivation of Zhejiang Sci-Tech University and Key Laboratory for RF Circuits and Systems (Hangzhou Dianzi University), Ministry of Education.

\section{Availability of data and materials}

Please contact author for data requests.

\section{Authors' contributions}

All authors take part in the discussion of the work described in this paper. These authors contributed equally to this work and should be considered co-first authors. Both authors read and approved the final manuscript.

\section{Competing interests}

The authors declare that they have no competing interests.

\section{Publisher's Note}

Springer Nature remains neutral with regard to jurisdictional claims in published maps and institutional affiliations.

\section{Author details}

'School of Information Science and Technology, Zhejiang Sci-Tech University, 928 Second Avenue, Xiasha Higher Education Zone, Hangzhou, Zhejiang, People's Republic of China. ${ }^{2}$ Key Laboratory for RF Circuits and Systems of Ministry of Education, Hangzhou Dianzi University, 1158, No. 2 Street, Xiasha Higher Education Zone, Hangzhou, Zhejiang Province 310018, People's Republic of China.

Received: 22 July 2018 Accepted: 28 September 2018 Published online: 24 October 2018

\section{References}

1. L.Q. Chen, Phase-field models for microstructure evolution. Annu. Rev. Mater. Res. 32(32), 113-140 (2002)

2. A.B. Bortz, M.H. Kalos, J.L. Lebowitz, A new algorithm for Monte Carlo simulation of Ising spin systems is. J. Comput. Phys. 17(1), 10-18 (1975)

3. H.W. Hesselbarth, I.R. Göbel, Simulation of recrystallization by cellular automata. Acta Metall. Mater. 39(9), 2135-2143 (1991)

4. Y. Liu, T. Baudin, R. Penelle, Simulation of normal grain growth by cellular automata. Scr. Mater. 34(11), 1679-1683 (1996)

5. J. Geiger, A. Roósz, P. Barkóczy, Simulation of grain coarsening in two dimensions by cellular-automaton. Acta Mater. 49(4), 623-629 (2001)

6. H.L. Ding, Y.Z. He, L.F. Liu, et al., Cellular automata simulation of grain growth in three dimensions based on the lowest-energy principle. J. Cryst. Growth 293(2), 489-497 (2006)

7. Y. He, H. Ding, L. Liu, et al., Computer simulation of $2 \mathrm{D}$ grain growth using a cellular automata model based on the lowest energy principle. Mater Sci Eng A 429(1), 236-246 (2006)

8. S. Raghavan, S.S. Sahay, Modeling the grain growth kinetics by cellular automaton. Mater Sci Eng A 445(6), 203-209 (2007)

9. W. Yu, Cellular Automata Modelling of Austenite Grain Coarsening during Reheating (University of Sheffield, 2002)

10. F. Hua, Y.S. Yang, D.Y. Guo, et al., Grain growth cellular automata model based on the curvature-driven mechanism. Acta Metall Sin 40(11), 1210-1214 (2004)

11. X.Y. Jiao, X.J. Guan, Y.T. Liu, et al., Simulation of grain growth based on Cellular Automata method[]]. Journal of Shandong University, 35(6), 24-28 (2005)

12. X.F. Ma, XJ. Guan, Y.T. Liu, et al., Cellular automaton model for grain growth based on modified transition rule[]. Chin. J. Nonferrous Metals. 18(1), 138-144 (2008).

13. M. Braginsky, V. Tikare, E. Olevsky, Numerical simulation of solid state sintering[J]. Int J Solids Struct 42(2), 621-636 (2005)

14. L. Nurminen, A. Kuronen, K. Kaski, Kinetic Monte Carlo simulation of nucleation on patterned substrates. Phys. Rev. B 63(3), 035407 (2000)
15. C.C. Battaile, D.J. Srolovitz, J.E. Butler, A kinetic Monte Carlo method for the atomic-scale simulation of chemical vapor deposition: application to diamond. J. Appl. Phys. 82(12), 6293-6300 (1997)

16. D. Raabe, L. Hantcherli, 2D cellular automaton simulation of the recrystallization texture of an IF sheet steel under consideration of Zener pinning. Comput. Mater. Sci. 34(4), 299-313 (2005)

17. M. Cook, Universality in Elementary Cellular Automata[]]. Complex Systems. 15(1), 1-40 (2004)

18. D. Spencer, Cities and Complexity: Understanding Cities with Cellular Automata, Agent-Based Models, and Fractals[J]. J. Reg. Sci. 83(2), 624-627 (2007)

19. A. Imre, G. Csaba, L. Ji, et al., Majority logic gate for magnetic quantum-dot cellular automata[J]. Science. 311(5758), 205-208 (2006)

20. J.I. Barredo, M. Kasanko, N. Mccormick, et al., Modelling dynamic spatial processes: simulation of urban future scenarios through cellular automata[]]. Landsc. Urban Plan. 64(3), 145-160 (2003)

21. J.J. Meléndez-MartíNez, A. DomíNguez-RodríGuez, F. Monteverde, et al., Characterisation and high temperature mechanical properties of zirconium boride-based materials. J. Eur. Ceram. Soc. 22(14), 2543-2549 (2002)

22. F. Monteverde, A. Bellosi, Effect of the addition of silicon nitride on sintering behaviour and microstructure of zirconium diboride. Scr. Mater. 46(3), 223-228 (2002)

23. L. Scatteia, D. Alfano, F. Monteverde, et al., Effect of the machining method on the catalycity and emissivity of $Z_{\mathrm{r}} B_{2}$ and $\mathrm{ZrB2}-\mathrm{HfB} 2$-based ceramics. J. Am. Ceram. Soc. 91(5), 1461-1468 (2010)

24. F. Monteverde, The addition of SiC particles into a MoSiz-doped ZrB2 matrix: effects on densification, microstructure and thermo-physical properties. Mater Chem Phys 113(2), 626-633 (2009)

\section{Submit your manuscript to a SpringerOpen ${ }^{\circ}$ journal and benefit from:}

- Convenient online submission

- Rigorous peer review

- Open access: articles freely available online

High visibility within the field

- Retaining the copyright to your article

Submit your next manuscript at $\boldsymbol{\nabla}$ springeropen.com 